\title{
Episodio Ilícito: Un Análisis de la Interferencia de EEUU en la Economía Colombiana (1989-2005)
}

\section{Illicit Episode: An Analysis of U.S. Interference in the Colombian Economy (1989- 2005)}

DOI: $10.46932 / \mathrm{sfjdv} 2 \mathrm{n} 2-124$

Received in: March 1st, 2021

Accepted in: May 30th, 2021

\author{
Osvaldo Alencar Billig \\ Maestría en Administración de Negocios, Especialización en Logística Empresarial, Licenciado en \\ Administración de Empresas. \\ Maestro del Centro Universitário Dinâmica das Cataratas - Foz do Iguaçu, Paraná, Brazil. \\ R. Castelo Branco, 440 - Centro, Foz do Iguaçu - PR \\ Correos: probillig@gmail.com
}

Bárbara Thayuska Lenz Rodrigues

Académico del curso de Relaciones Internacionales en el Centro Universitário Dinâmica das Cataratas. Endereço: Rua Uruguai, 836 - Centro, Entre Rios do Oeste, PR. Brazil.

Correos: barbaralenz25@outlook.com

\section{RESUMEN}

En el escenario internacional de consumo ilícito y tráfico de drogas exacerbado entre la década de 1970 y el comienzo del siglo XXI, los Estados Unidos de América adoptaron medidas diplomáticas, económicas y militares intervencionistas como justificación para garantizar su seguridad nacional y contener el avance de la ideología comunista en América Latina, donde Colombia fue uno de los principales actores de este episodio estadounidense. El objetivo de este artículo es dilucidar la interferencia directa del poder capitalista estadounidense en los territorios latinoamericanos y especialmente en Colombia, para dar fe de que los EE.UU. fueron el gran pivote de las crisis económicas y sociales en Colombia en ese período. La metodología utilizada para esta labor fue la investigación, el estudio y la revisión bibliográfica de artículos publicados en revistas, periódicos, libros y otras plataformas digitales. En resumen, fue posible identificar que las medidas neoliberales de interferencia de los Estados Unidos en el contexto colombiano a finales de la década de 1980 desencadenaron el detrimento económico y social en el país hasta principios de la década de 2000.

Palabras clave: narcotráfico, Colombia, América Latina, Estados Unidos.

\begin{abstract}
In the international scenario of illicit consumption and drug trafficking exacerbated between the 1970s and the beginning of the 21st century, the United States of America adopted interventionist diplomatic, economic and military measures as a justification to guarantee its national security and to contain the advance of communist ideology in Latin America, where Colombia was one of the main actors in this U.S. episode. The aim of this article is to elucidate the direct interference of US capitalist power in Latin American territories and especially in Colombia, to attest that the US was the great pivot of the economic and social crises in Colombia in that period. The methodology used for this work was the research, study and bibliographic review of articles published in magazines, newspapers, books and other digital platforms. In summary, it was possible to identify that the neoliberal interference measures of the United
\end{abstract}


States in the Colombian context in the late 1980s triggered the economic and social detriment in the country until the early 2000 s.

Keywords: drug trafficking, Colombia, Latin America, United States.

\section{INTRODUCCIÓN}

En los decenios de 1970 y 1980 la población mundial experimentó un aumento más significativo del consumo de drogas ilícitas, especialmente de marihuana y cocaína, en suelos europeos y americanos. Los cárteles ilegales para la producción y el tráfico internacional de estas drogas han crecido hasta convertirse en un negocio muy lucrativo en América Latina. Colombia, conocida por sus mayores cultivos de coca y producción de cocaína en el mundo, es sede de varias redes de tráfico de drogas, especialmente en los Estados Unidos de América, donde la mayor parte de la sustancia ilícita que se comercia es de origen colombiano.

En los Estados Unidos, Ronald Reagan, presidente de los Estados Unidos (1981-1989), estableció una intensa política de lucha contra el tráfico de drogas en su territorio y en América Latina, con la justificación de que el tráfico de drogas se había convertido en una preocupación internacional y una amenaza para la seguridad regional de los Estados Unidos. Por ejemplo, en el gobierno de Reagan, gran parte de los recursos financieros se destinaron a la guerra contra las drogas, además de la movilización de las Fuerzas Armadas de los Estados Unidos para actuar en suelo extranjero en este combate, y el uso de la diplomacia de represalias (aplicación de sanciones a los países objetivo). Más tarde, Bill Clinton, durante su mandato como presidente de los Estados Unidos (1993-2001), continuó la lucha contra la producción y comercialización de drogas en América, pero, a diferencia de Reagan, Clinton adoptó políticas de responsabilidad compartida en lugar de la militarización, lo que supuso una reacción más positiva en la lucha contra el narcotráfico.

El tema que retratará la Iniciativa Andina y la implementación del Plan Colombia, ambos marcados por la injerencia de Estados Unidos en el país latino entre 1989 y 2005, fue escogido, analizado y estudiado en este artículo por sus propuestas de apertura de los mercados para la penetración del capitalismo estadounidense frente a la ideología comunista y la lucha contra el narcotráfico en Colombia, respectivamente, y por la dificultad de lograr el objetivo principal: acabar con las plantaciones de coca, la amapola sativa de la región y, consecuentemente, con la producción de cocaína. La Iniciativa Andina, por lo tanto, al dar cabida a los competidores de las exportaciones para que entren en Colombia, ha intensificado una crisis económica nacional y ha aumentado la agricultura ilícita. El Plan Colombia, a su vez, se puso en práctica con el apoyo de los Estados Unidos, que además de participar con recursos económicos en esta iniciativa, tenían interés en erradicar el tráfico de cocaína de Colombia a su territorio. 
Sin embargo, además de causar graves problemas ambientales que amenazan la salud de los colombianos, el Plan tampoco logró los resultados esperados.

La metodología utilizada para la realización y estudio de este artículo fue la investigación y revisión bibliográfica de obras ya publicadas por otros investigadores, autores y estudiosos de la materia y correlaciones, mediante artículos científicos, investigaciones documentales, libros, artículos en revistas nacionales e internacionales y periódicos digitales. Inicialmente, se hizo la investigación y la elección de la bibliografía, con el fin de encontrar estudios y obras que pudieran orientar, apoyar y certificar mejor la construcción de este artículo. Posteriormente, se leyeron y archivaron los materiales elegidos, sintetizando las ideas para delimitar el objetivo del artículo. Finalmente, se redactó y elaboró el trabajo en base y con la contribución de todos los autores referenciados, concluyendo así el análisis de la interferencia estadounidense y sus consecuencias para la economía colombiana en el contexto abordado, logrando el objetivo propuesto.

La importancia del tema estudiado va más allá de la propuesta de lucha contra las drogas, pero saca a la luz el contexto que llevó a Colombia a convertirse en este centro de producción y comercialización de estupefacientes, ya que incluso la injerencia de Estados Unidos en América Latina en la guerra contra las drogas y la financiación del Plan Colombia, fueron las consecuencias de las políticas económicas estadounidenses impuestas a los países latinoamericanos a finales de la década de 1980 las que llevaron al país colombiano a intensificar el cultivo y el tráfico internacional de sustancias ilícitas, como se expondrá en este trabajo.

\section{ENTORNO DE INTERFERENCIA}

En el decenio de 1970 se produjo por primera vez en el mundo un aumento de la producción y el consumo de drogas, principalmente por parte de los países europeos y los Estados Unidos, que, al adoptar medidas nacionales para combatir el cultivo, el comercio y el uso de sustancias ilícitas de manera rígida, abrieron indirectamente sus puertas a la entrada ilegal de estupefacientes procedentes del tráfico internacional. La cocaína estaba ganando terreno en los Estados Unidos y afectaba cada vez más a los consumidores muy jóvenes, que gradualmente empezaron a consumir sustancias psicoactivas (FRAGA, 2007). A medida que este aumento continuaba en el decenio de 1980, las políticas represivas de los Estados Unidos en materia de narcotráfico se hicieron más importantes y tuvieron un lugar urgente en los intereses políticos de los gobiernos, llamándose guerra contra las drogas.

En la esfera social americana se arraigó la moral puritana, con una ética basada en el individualismo y el protestantismo de dedicación al trabajo, lo que provocó una reacción de repudio a la adicción y a la dependencia química de los narcóticos. Este repudio llevó a la criminalización por parte 
del Estado de la producción, venta y consumo de marihuana, cocaína, heroína, opio, entre otras drogas en el territorio nacional. Además, cuando se planteó esta cuestión en el sistema internacional, los Estados Unidos no escatimaron esfuerzos militares ni recursos financieros para poner fin al consumo y el tráfico ilícito de estupefacientes dentro de sus fronteras (SANTOS, 2006).

Como principal potencia capitalista mundial desde el final de la Segunda Guerra Mundial (19391945), los Estados Unidos tenían un papel decisivo en la organización del actual orden económico y político internacional, y para establecer su hegemonía como sistema económico dominante, el Estado de los Estados Unidos tenía que difundir sus políticas monetarias y económicas a los países de América Latina, donde podría actuar e integrar a otras naciones americanas en su propio beneficio (SANTOS, 2006). Desde que la Unión de Repúblicas Socialistas Soviéticas (URSS) se había consolidado como potencia militar en el contexto de la Guerra Fría (1945-1991), el objetivo de los Estados Unidos era contener la amenaza comunista de manera universalizada y, basándose en políticas y acciones represivas internas al consumo y tráfico de estupefacientes, los Estados Unidos también llevaron la diplomacia de la lucha contra el tráfico de estupefacientes al escenario internacional, distorsionando y manipulando la imagen de la URSS como responsable del narcoterrorismo.

Temiendo el auge de las políticas ideológicas de la Unión Soviética sobre las naciones latinas y empezando por la Iniciativa Andina, los Estados Unidos aprovecharon la oportunidad para reafirmar su autonomía militar y económica en América Latina en conjunción con su diplomacia de la guerra contra las drogas, utilizando un discurso de "víctima" en relación con los grupos extranjeros de narcotraficantes y alegando una amenaza a su seguridad nacional, asociando este narcotráfico exacerbado con el terrorismo y el auge de las ideologías comunistas. Así, Estados Unidos intentó, a través de políticas económicas liberales, impulsar su capitalismo cerca de los países latinoamericanos, con el fin de reconectar y consolidar su dominio ideológico, político y económico en la región (RODRIGUES, 2012). Sin embargo, aun argumentando que su intervención andina traería buenos resultados económicos a sus países hermanos de América Latina, la realidad de Colombia después de la imposición de las medidas fue un desastre.

En vista de ello, con la disminución de la producción de drogas en suelo estadounidense y el aumento del consumo de sustancias ilícitas en los Estados Unidos, el comercio internacional ilegal de narcóticos de países latinos, como Colombia, ha crecido rápidamente para atender las demandas de los países del centro capitalista. Sin embargo, además de la demanda del consumo de drogas, este comercio ilegítimo pretendía hacer frente a la crisis económica interna colombiana, consecuencia de varios factores internos y externos, pero que tuvo como una de sus mayores influencias las reformas neoliberales de la Iniciativa Andina, proyecto de intervención llevado a cabo por uno de los presidentes de Estados Unidos en 1989 (RIPPEL, 2006). 


\subsection{La Iniciativa Andina y el "Efecto Globo"}

América Latina y otras regiones periféricas de Asia, en las últimas décadas del siglo XX, fueron la sede de la división internacional de cultivo, producción y comercialización de drogas ilegales, siendo estos países los mayores proveedores de drogas para atender los mercados de las naciones del centro (SANTANA, 1999). Con el aumento de la demanda de sustancias ilícitas en los Estados Unidos y el contexto de inestabilidad económica establecido en la región de América Latina en los decenios de 1970 y 1980, resultante de las políticas liberales de los Estados Unidos, países como Colombia, el Perú y Bolivia encontraron en los cultivos de adormidera, coca y cannabis sativa una salida para hacer frente a las crisis financieras, abasteciendo el mercado internacional de drogas. Asociada a la necesidad de combatir sus crisis internas, la producción de drogas en América Latina refleja también una dependencia económica de esta región respecto de los Estados Unidos, resultado de su capitalismo.

Situada en Sudamérica, Colombia es una república presidencial con una población total de 49.084.841 habitantes (CIA, 2020), con el español como lengua oficial. Se independizó de España el 20 de julio de 1810, pero aun así, desde su emancipación, la nación colombiana ha sido gobernada por una oligarquía opresora que no ha desarrollado reformas estructurales en las esferas sociales y económicas del país. Además, Colombia siempre ha vivido con gran dificultad e incapacidad para consolidar su unidad nacional con el fin de garantizar su soberanía y el funcionamiento efectivo del Estado (SANTOS, 2010). Estas inconsistencias internas, de crisis institucional y económica, han creado condiciones para la plantación de coca, atrayendo a varios agricultores a cultivar esta planta como una forma de supervivencia. En relación con el inestable contexto nacional, los cambios e imposiciones económicas de los Estados Unidos han fomentado el declive financiero de Colombia y el crecimiento de la producción de estupefacientes en el país.

Con el objetivo de consolidar su hegemonía en el continente americano y en un intento de poner fin a los problemas del uso y el comercio ilegal de estupefacientes, los Estados Unidos adoptaron medidas para combatir esta delincuencia organizada transnacional y, en 1973, durante el mandato presidencial de Richard Nixon (1969-1974), se creó la Drug Enforcement Agency Administration (DEA) para reforzar su aparato de represión contra los estupefacientes, con la función de ocuparse de la política nacional antidroga y de las acciones contra el tráfico de estupefacientes en el extranjero. Más tarde, el presidente estadounidense Ronald Reagan (1980-1988) definió en una directiva el problema de la "guerra contra las drogas" como un asunto de seguridad nacional, y desde su administración, Estados Unidos ha intensificado su política antidroga, adoptando como medida el fin de la producción de drogas en países como Bolivia y Perú (SILVA, 2015). Esta política de Reagan, acordada por la diplomacia estadounidense en la lucha contra el "narcoterrorismo", aprobó en 1982 la Ley de Autorización de la Defensa, que permitió 
al ejército estadounidense actuar para combatir la producción, el comercio y el consumo de narcóticos, reflejando la fuerte militarización de las políticas antidrogas del gobierno de Reagan (SANTOS, 2010).

Sin embargo, en 1989 el presidente de los Estados Unidos George H.W. Bush, al asumir su mandato presidencial, anunció la Iniciativa Andina como un plan para desarrollar el comercio exterior de los países andinos a través de incentivos financieros y financiación de los Estados Unidos. Estas reformas neoliberales implementadas en Colombia han obligado al país a abrir su economía y sus mercados al capitalismo estadounidense, lo que ha traído un crecimiento en el flujo de inversión extranjera y de bienes hacia el territorio colombiano. Con posterioridad a esta apertura, la balanza comercial de Colombia mostró tasas de déficit, ya que esta política económica afectó sustancialmente a los agricultores, campesinos y productores nacionales, que no estaban en condiciones de competir con las grandes industrias de alta tecnología de las naciones desarrolladas (RIPPEL, 2006).

En el contexto del déficit de exportación, además de los artículos industrializados producidos por Colombia, varios productos agrícolas colombianos, como el café y el tabaco, han sufrido demasiado por la competencia externa, lo que ha llevado a los productores a sustituir sus cultivos por la siembra de coca y amapola, materias primas de la cocaína y la heroína respectivamente. Para ilustrar esta competencia, en 1984 sólo el 15 por ciento de los cigarrillos vendidos en Colombia eran importados, pero en 1993 ese porcentaje se elevó al 70 por ciento. El mismo problema se puede observar en relación con el café que, con la entrada de nuevos competidores y productores de este producto agrícola en el mercado internacional, provocó una fuerte caída del precio del grano en la producción nacional colombiana (RIPPEL, 2006).

Con la crisis financiera resultante de las intervenciones económicas de los Estados Unidos, en 1999 se produjo la peor recesión económica de los últimos cincuenta años a la que tuvo que hacer frente Colombia y, junto con ella, la tasa de desempleo alcanzó un hito de casi el 20\% en el país (RIPPEL, 2006). En un intento por reconstruir su economía, en diciembre del mismo año el gobierno colombiano contrajo un préstamo de 2.700 millones de dólares del Fondo Monetario Internacional (FMI), siendo una de las condiciones para el otorgamiento de este crédito una mayor apertura de la economía nacional a favor del capital extranjero.

Este clima económico de crisis en Colombia (consecuencia del neoliberalismo estadounidense), junto con las políticas de exterminio de las plantaciones de coca en el Perú y Bolivia por la militarización estadounidense en la lucha contra el narcotráfico, llevó a varios productores rurales colombianos a cultivar la materia prima de la cocaína, aumentando enormemente una producción ya existente. Por un lado, como alternativa a la competencia de los productos importados, para que éstos tengan un mayor rendimiento financiero; y por otro, el "efecto globo" provocado por la migración de estos cultivos desde los suelos 
peruanos y bolivianos hacia el territorio colombiano, donde hasta entonces no se había producido un proceso tan intenso de erradicación de la producción de drogas ilícitas (RIPPEL, 2006; SANTOS, 2006).

La producción y comercialización ilegal de marihuana, heroína y cocaína (principalmente), que a partir de entonces se produjo en gran escala, entró en el territorio de los Estados Unidos (como principal comprador y consumidor de drogas) para satisfacer la gran demanda de los estadounidenses. Este nuevo proveedor latino causó más preocupación al gobierno de Estados Unidos, como otra amenaza a su seguridad nacional y a su diplomacia del narcotráfico. Por lo tanto, para el gobierno de los Estados Unidos, como de costumbre, era el momento de intervenir (CAIXETA, 2018; RIPPEL, 2006).

\section{PLAN DE COLOMBIA (1999-2005)}

El alto consumo de drogas en los Estados Unidos, el inestable escenario económico y social de Colombia y el aumento del cultivo de cocaína, heroína y materias primas de marihuana en Colombia han alentado las iniciativas del gobierno colombiano de Andrés Pastrana (1998-2002) para crear una estrategia de lucha contra estas adversidades en su estado, Pues aunque ya se estaban utilizando herbicidas para destruir las plantaciones de coca, adormidera y cannabis sativa, estas medidas no bastaban para erradicar esta producción y perjudicaban directamente a los trabajadores rurales, al medio ambiente y a otras plantaciones legales, causando un daño incalculable en el territorio nacional del país latino (FRAGA, 2007); SANTOS, 2006).

El presidente colombiano pretendía recuperar la confianza de su población mediante el restablecimiento de las normas sociales básicas, el respeto de los derechos humanos y el mantenimiento del orden público, así como la promoción de la integridad territorial, la justicia y la democracia, a través de proyectos que beneficien a los colombianos menos favorecidos. Así, en 1998, el gobierno de Pastrana creó el Plan Colombia (PC) como una estrategia gubernamental para la paz, la prosperidad y el fortalecimiento del Estado colombiano (RIPPEL, 2006). Además, para combatir la violencia dentro de su país, Pastrana se unió a su apoyo político interno para iniciar conversaciones de paz con las Fuerzas Armadas Revolucionarias de Colombia (FARC), siendo uno de sus primeros pasos la creación de una zona desmilitarizada en 1999, donde no había ni policía ni militares, con el objetivo de acercarse al grupo revolucionario (SILVA, 2015). Además de estas estrategias gubernamentales, también existía un programa de sustitución de los cultivos de drogas por cultivos alternativos, con la intención de no perjudicar a los agricultores y productores rurales que veían exterminados sus cultivos por el exterminio de estos cultivos ilícitos (SANTOS, 2006).

Con el fin de aprovechar su estrategia de gobierno, Pastrana buscó financiación internacional para el Plan Colombia. Así pues, presentó el CP al gobierno de los Estados Unidos, que también estaba muy 
interesado en erradicar el tráfico de drogas en su país y creía que los países latinos, especialmente Colombia, no tenían las condiciones para resolver los problemas de la producción y el tráfico internacional de drogas a través de sus propias instituciones nacionales. El Plan Colombia fue aprobado entonces por el Congreso de los Estados Unidos y por el presidente Bill Clinton, pero sólo con la condición de que elaborara una estrategia bien definida de lucha contra el narcotráfico, con un fuerte carácter policial y militar. El gobierno colombiano aceptó las condiciones y adaptó su estrategia para recibir apoyo financiero de los EE.UU. Así, el Plan Colombia tenía un total de 7.500 millones de dólares para ser financiado, con la mayoría de los recursos provenientes del gobierno de los Estados Unidos, pero también contando con contribuciones de Europa y la comunidad internacional, desde 1999 hasta 2005. Sin embargo, este carácter bélico y represivo adoptado por Colombia para combatir el narcotráfico hizo que las FARC se descontentasen y asumiesen el control de la zona militarizada pacífica que había creado Pastrana y fomentase un aumento de la violencia y los índices de homicidios en el país (RIPPEL, 2006; SILVA, 2015). El gobierno colombiano, entonces, parte para el punto principal de su estrategia: acabar con el cultivo de materias primas para la producción de drogas en el país.

La fumigación con el uso de herbicidas cobra más fuerza y obliga a las personas que cultivaban cultivos de coca, adormidera y cannabis sativa a erradicar estos cultivos a cambio de una compensación pagada en efectivo por hectárea y también por cultivos alternativos como plátanos, palmitos, pimienta, flores tropicales, cacao y maíz. Sin embargo, estos productos utilizados para sustituir los cultivos exterminados no tenían el mismo poder competitivo en los mercados nacionales e internacionales, lo que los hacía económicamente inviables para los pocos productores rurales que recibían esta asistencia y, además del daño económico que tenían estos productores, los herbicidas aplicados a la tierra para acabar con las plantaciones dejaban graves consecuencias y riesgos para la salud de los colombianos y dañaban demasiado el medio ambiente (FRAGA, 2007; SANTOS, 2006). Además, este exterminio de las plantaciones de coca, amapola y cannabis sativa en el territorio colombiano ha desencadenado un nuevo "efecto globo" con sus vecinos, teniendo repercusiones negligentes con los países de Bolivia y Perú, que han sido testigos de un aumento de los cultivos de coca como nunca antes se había producido en sus tierras nacionales (SILVA, 2015).

Estados Unidos, al vislumbrar los efectos negativos del Plan Colombia, con la no disminución del consumo de drogas en su país, el aumento de la inestabilidad económica y social dentro del país colombiano, debido principalmente al gran incremento de la violencia y las violaciones de los derechos humanos, y la migración de estos cultivos exterminados a los suelos de los países vecinos, se dio cuenta de que el PC no alcanzaba los objetivos estipulados, sino que por el contrario, fracasaba y perjudicaba a otros países. Además, el gobierno de Bill Clinton también analiza que una de las razones por las que el 
Plan Colombia no es suficiente para lograr sus objetivos son los problemas estructurales internos de Colombia, ya mencionados aquí. Sin embargo, aun así, el gobierno de los Estados Unidos, a partir de ahora bajo el mandato de George W. Bush (2001-2009), preocupado por su seguridad nacional, preocupación que se ve acrecentada por el ataque terrorista en la ciudad de Nueva York el 11 de septiembre de 2001, decide crear otra iniciativa para continuar el Plan Colombia (SANTOS, 2006).

Con la nueva doctrina antiterrorista de la administración Bush tras el episodio del 11 de septiembre y la elección de Álvaro Uribe Vélez como presidente de Colombia (2002-2006), el foco del PC gana una expansión. Los presidentes de Estados Unidos y Colombia coinciden en que la idea de que los grupos terroristas que controlan el narcotráfico y amenazan la seguridad nacional e internacional son las guerrillas. Así, con objetivos propios, pero con intereses comunes, la Iniciativa Regional Andina que se creó en 2001 y que se rebautizó como Iniciativa Andina Antidrogas en 2003, surge como una continuación del Plan Colombia para acabar con el narcotráfico, en el que la administración Bush aumentó los recursos económicos de los países vecinos para combatir también el narcoterrorismo (SANTOS, 2006; SANTOS, 2010).

Con cinco años de vigencia del Plan Colombia, los datos de los gobiernos de Colombia y Estados Unidos mostraron una reducción en la producción de cocaína de 617 toneladas en 2001 a 390 toneladas a principios de 2005, un total de 36\% de una meta de por lo menos 50\% de esa reducción. En relación con el cultivo de la hoja de coca, hubo una reducción de 180.000 hectáreas plantadas en 2001 a 65.000 hectáreas plantadas en 2005. Sin embargo, según la Organización de las Naciones Unidas (ONU), la tasa de replantación de esta hoja es alta en las zonas de erradicación, llegando al 40\%, lo que hace que los datos de las reducciones mencionadas sean desalentadores. Además, según el Informe Mundial sobre las Drogas, transmitido por las Naciones Unidas en 2005, Colombia seguía siendo el mayor productor mundial de cocaína, con un 56\% del total, lo que demuestra claramente el fracaso de esta estrategia.

\section{OBSERVACIONES FINALES}

El contexto de inestabilidad económica y social en Colombia entre 1989 y 2005 es consecuencia de varios factores internos y externos, pero sin duda la interferencia neoliberal y represiva de los gobiernos de los Estados Unidos a lo largo de este período fue fundamental para establecer este escenario en el país latino. A medida que estas intervenciones de los Estados Unidos se desarrollaban en la vida colombiana, los acontecimientos podían verse de manera trágica, como se explica en este documento, con la profundización de la crisis económica, el aumento de la tasa de violencia y la violación de los derechos humanos de la población, la alta tasa de desempleo y la dificultad de contener las revueltas armadas dentro del territorio nacional, siendo esta imposición de los Estados Unidos un objetivo de su propio interés 
nacional en defensa de su seguridad regional y su consolidación como potencia ideológica y económica sobre América Latina.

La Iniciativa Andina, cuyo objetivo era abrir los mercados latinos al capitalismo, desencadenó el comercio internacional con proveedores que sustituyeron a los productores rurales colombianos, que no tenían la capacidad de competir con estos nuevos actores presentes en sus redes comerciales. Y junto con esta circunstancia, el "efecto globo" de la migración de los cultivos de coca, amapola y cannabis sativa peruanos y bolivianos a Colombia, llevó a estos mismos productores afectados por las medidas neoliberales de los Estados Unidos a cultivar estos cultivos, como una forma de sobrevivir y buscar un retorno financiero.

Los intentos de poner fin al cultivo de cocaína (principalmente), heroína y materias primas de marihuana en suelo colombiano para acabar con el tráfico de estos estupefacientes dentro de las fronteras de Estados Unidos no fueron suficientes para que esto ocurriera, sino que, por el contrario, desestabilizaron una economía y una sociedad que ya había traído problemas estructurales desde su independencia. Las opresivas medidas policiales y militares adoptadas desencadenaron las revueltas de las FARC en el país, y la falta de apoyo a los agricultores a los que se exterminaron sus cultivos provocó una inestabilidad aún mayor.

Los datos presentados al final del propio Plan Colombia indican que el objetivo de eliminar las plantaciones y acabar con el narcotráfico en la región de América Latina y especialmente en los Estados Unidos ha fracasado de manera significativa y ha causado también efectos desastrosos para la población colombiana, además de afectar de manera negligente a sus países vecinos.

El propósito de todas estas intervenciones e intentos de cambio siempre ha estado vinculado a la economía de los Estados Unidos y a la imposición capitalista. Sin embargo, aunque Colombia estuviera pasando por este mismo contexto, las estrategias a implementar deberían tener en cuenta el problema real: las desigualdades sociales y políticas de este país, porque como ya se ha mencionado, la nación latina no había pasado por reformas estructurales. Si tanto el gobierno colombiano como el estadounidense se hubieran apartado de este punto, el problema podría resolverse desde dentro hacia fuera, centrándose en quién se verá realmente afectado por estos cambios: la sociedad colombiana. 


\section{REFERENCIAS}

AGENCIA CENTRAL DE INTELIGENCIA (2020) El World Factbook - América del Sur: Colombia. https://www.cia.gov/library/publications/the-world-factbook/geos/co.html. Acessado em 19 de setembro de 2020.

CAIXETA, Thayse Cristine (2018) El tráfico de drogas en Colombia y la aplicación del Plan Colombia a principios de 2000. Trabajo de conclusión del curso (Graduación en Relaciones Internacionales) Universidade Federal de Uberlândia, Uberlândia, 2018. http://clyde.dr.ufu.br/handle/123456789/22112. Acessado em 03 de setembro de 2020.

FRAGA, Paulo César Pontes (2007) La geopolítica de las drogas en América Latina. Revista da Faculdade de Serviço Social do Estado do Rio de Janeiro, n. 19. https://www.epublicacoes.uerj.br/index.php/revistaempauta/article/view/187. Acessado em 03 de setembro de 2020.

RIPPEL, Márcio Pereira (2006) El Plan Colombia como instrumento de la política americana para América Latina y sus consecuencias. Revista da Escola de Guerra Naval, edición portuguesa; Río de $\begin{array}{lllll}\text { Janeiro } & \text { Vol. } & 7, & \text { (2006): }\end{array}$ https://search.proquest.com/openview/dc7912aa3bc5b90be364bb46c581396f/1?pqorigsite=gscholar\&cb l=4457491. Acessado em 19 de setembro de 2020.

RODRIGUES, Thiago (2012) El narcotráfico y la militarización en América: la adicción a la guerra. Contexto Int. Rio de Janeiro, v. 34, n. 1, p. 9-41, junio de 2012. http://www.scielo.br/scielo.php?script=sci_arttext\&pid=S010285292012000100001\&lng=en\&nrm=iso. Acessado em 19 de setembro de 2020.

SANTANA, Adalberto (1999) Una globalización del tráfico de drogas. Rev. bras. polít. int., Brasília, v. 42, n. $2, \quad$ p. 1999. http://www.scielo.br/scielo.php?script=sci_arttext\&pid=S00347329199900020006\&lng=en\&nrm=iso. Acessado em 21 de novembro de 2020.

SANTOS, Marcelo (2006) La política de los Estados Unidos contra el narcotráfico y el Plan Colombia (1998-2005). Estudios de Sociología, Araraquara, v. 12, n. 22, p. 169-188, 2006. https://periodicos.fclar.unesp.br/estudos/article/view/354 Acessado em 20 de setembro de 2020.

SANTOS, Marcelo (2010) Pasado y presente de las relaciones entre Colombia y Estados Unidos: la estrategia de internacionalización del conflicto armado colombiano y los lineamientos de la política exterior estadounidense. Rev. bras. polít. int. Brasilia, v. 53, n. 1, p. 67-88, Jul. 2010. http://www.scielo.br/scielo.php?script=sci_arttext\&pid=S0034-73292010000100004\&lng=en\&nrm=iso. Acessado em 09 de novembro de 2020.

SILVA, Caroline Cordeiro Viana (2015) COLOMBIA PLAN: Securitización del tráfico de drogas por los Estados Unidos de América. Conjuntura Global, v. 4, n. 2, mayo, 2015, p. 124-132. https://revistas.ufpr.br/conjgloblal/article/view/43167/26164. Acessado em 21 de novembro de 2020. 